\title{
Eine neue Niphargus-Art (Gammaridae, Amphipoda) aus Bulgarien
}

\author{
Von Stoitze Andreev ${ }^{1}$ ) \\ Mit Tafel $53(1)-54$ (2) \\ Niphargus toplicensis n. sp.
}

Körper des Männchens schlank und langgestreckt, des Weibchens kürzer und breiter. Farbe weiß bis milchiggelb. Augen fehlen. Körperlänge des $\widehat{\jmath} 8 \mathrm{~mm}$, des + mit gut entwickelten Oostegiten bis $7 \mathrm{~mm}$.

I. Antenne verhältnismäßig lang, erreicht bei beiden Geschlechtern 2/3 der Körperlänge. Geißel des ơ 22-26gliedrig, des 우 18-21gliedrig. Nebengeißel 2gliedrig, länger als das I. Glied. Hyaline Stäbchen so lang oder ein wenig kürzer als die halbe Länge des entsprechenden Gliedes. II. Antenne übertrifft die halbe Länge der I. Antenne, mit einer 8-10gliedrigen Geißel.

Mandibel mit gut entwickelter Kaufläche (Abb. 1). II. und III. Palpusglieder gleichlang. Innenrand des III. Gliedes mit einer Reihe kurzer Borsten, apikal mit 6-7 längeren inseriert.

Innenlade der I. Maxille kurz, mit 3, öfter 4, nicht langen Stacheln versehen (Abb. 2). Außenlade mit 7 Stacheln, der innere mit 3-4 feinen Seitenzähnen: bei dem zweiten nur selten 2 Seitenzähne, die übrigen bloß mit einem stark entwickelten Seitenzahn. An der Spitze des Palpusgliedes befinden sich 5-6 Borsten so lang wie das Palpusglied. An der Innenseite des basalen Palpusgliedes gibt es 1 oder 2 Borsten, fast so lang wie das Palpusglied.

Innenlade des Maxillarfußes (Abb. 3) erreicht den äußeren distalen Rand des basalen Palpusgliedes. An dessen Spitze befinden sich 4-5 glatte meißelartige Stacheln und 4-5 Borsten. Außenlade erreicht fast die Hälfte des II. Palpusgliedes.

Coxalplatten länger als breit, überholen die entsprechenden Segmente. IV. Coxalplatte des đิ am größten und fast quadratisch. V., VI. und VII. Coxalplatte bedeutend kleiner als die übrigen.

1) Institut Zoologique et Musée de l'Académie des Sciences de Bulgarie, 1 Boulevard Ruski, Sofia. 
Gnathopoden (Abb. 4, 5) groß, fast quadratisch, etwas länger als breit. I. Gnathopod kleiner als der II. Carpen gut entwickelt. Palma etwas schräggestellt, etwas länger oder gleichlang wie der hintere Rand des Propodus. Es ist von einem stark entwickelten Rand begrenzt, aus dessen Grund 1-2 feingezähnte Stacheln hervorragen. Der hintere Rand der Gnathopoden des $q$ mit 5-7 Borstengruppen und des ô mit 9-10 versehen. In der Nähe des vorderen Randes befinden sich 1-2 Gruppen mit je 3-5 langen Borsten. Rückenseite der Dactyli mit je 5-6 Borsten versehen. An der inneren Seite im Grunde der Klaue ein gut entwickelter Stachel.

Pereiopoden mit verhältnismäßig breiten Basen mit konvexen Vorder- und schwachen konkaven Hinterrändern. VII. Pereiopod (Abb. 6) überragt die halbe Körperlänge. Klaue des Dactylus stark entwickelt und erreicht $2 / 3$ von dessen Länge. Aus seinem Grunde ragen ein starker Stachel und eine Borste hervor. Eine befiederte Borste befindet sich auch auf dem Rücken des Dactylus. II. und III. Epimeren (Abb. 7) mit fast rechtwinkligen, scharf ausgezogenen Ecken. Hinterrand des I. Epimers nicht scharf ausgezogen, schwach konvex. Unterrand des II. Epimers mit 2, jener des III. mit 3 starken Stacheln.

Pleopoden mit 2 Retinakula (Abb. 8).

Beim I. Urosomsegment im Grunde des I. Uropoden ein starker Stachel; II. Urosomsegment dorsal jederseits mit 1 Borste; III. Urosomsegment mit einem starken Stachel und einer Borste. I. Uropod des $\widehat{o}$ (Abb. 9) mit Innenast 2mal so lang wie der äußere, die beiden länger als das Basalglied. Beim $q^{1 / 5}$ bis $\mathbf{1} / \mathbf{4}$ länger als der innere. Innenast des II. Uropoden (Abb. 10) etwas länger oder so lang wie der äußere. III. Uropod (Abb. 11) des ô differenziert, das II. Glied des Außenastes 1/4 kürzer als das I. Glied. Innenast spießartig, verlängert. III. Uropod des $q$ nicht differenziert mit kurzem II. Glied am Außenast und mit kleinem schuppenartigem Endopodit.

Telson (Abb. 13) breiter als lang, etwa $2 / 3$ gespalten, verhältnismäßig weit klaffend, an der Spitze mit 3-4, selten mit 5 starken Stacheln (nicht lang) versehen.

Auf dem Rücken marginal mit einer Sinnesborste, bei manchen Exemplaren mit einem starken Stachel.

Durch seinen schlanken und ausgezogenen Körper, durch die Ausbildung der Gnathopoden und den langen II. Uropod zeigt N. toplicensis eine Ähnlichkeit mit einigen dem $N$. aquilex verwandten Arten, die in Südosteuropa verbreitet sind, wie z. B. $N$. aquilex moldavicus Dobreanu, Manolache, Puscariu, 1953, N. smederevanus Karaman, 1950, N. anatolicus Karaman, 1950, N. pancici Karaman, 1929, N. pancici olkanovi Karaman, 1959. 
Von $N$. aquilex moldavicus weicht $N$. toplicensis durch die Aufrüstung der I. Maxille, den Maxillarfuß, die Ausbildung der Gnathopoden, die längeren I.-IV. Coxalplatten, die Epimeren u.a. ab.

Wenn auch dem N. smederesanus gleichend, kann $N$. toplicensis nicht zu dieser Art gezählt werden, denn sie unterscheidet sich durch 4 Borsten an der Innenlade der I. Maxille, durch die stark entwickelte Kaufläche der Mandibel, durch die mehreren glatten und meißelartigen Stacheln an der Innenlade des Maxillarfußes, die I.-IV. Coxalplatten, die länger als breit sind, durch die Dactyli der IV., V. und VI. Pereiopoden mit je einem Stachel. Telson bei unserer Art ist breiter als lang, ohne die reiche Aufrüstung auf dem Rücken, charakteristisch für smederevanus.

Dies ist auch der Fall bei $N$. anatolicus, der sich von toplicensis durch die I.-IV. Coxalplatten, die länger als breit sind, durch die Aufrüstung der I. Maxille, den Maxillarfuß und die stark gerundeten Epimeren unterscheidet.

$N$. toplicensis steht mittels mehrerer Merkmale der von Karaman beschriebenen Art $N$. pancici und besonders der Unterart aus Bulgarien $N$. p. slkanovi sehr nahe, weicht aber durch die scharf endenden II. und III. Epimeren, I. Uropod, mehrere Borsten an der I. Maxille, Telson u.a. ab. Näher verwandt ist toplicensis mit der Unterart slkanovi, doch auch dazu kann sie nicht gezählt werden wegen der reichen Bestachelung der Innenlade der I. Maxille, der längeren als breiten Coxalplatten, nicht so schlanken Pereiopoden, II. und III. Epimeren. Während bei $N$. p. vlkanovi am Dactylus des VII. Pereiopoden nur ein Zahn am Grunde der Klaue steht, erscheint bei toplicensis auch eine Borste, wie auch an der Außenseite des Dactylus eine Sinnesborste.

N.toplicensis wurde von V. Guéorguiev in der Quelle,,Toplitzata" bei dem Dorf Mussomischta (Bezirk Goze Delcev) am 21. Mai 1962 gefunden.

Es wurden 10 ô, 7 우 und 14 juvenile Exemplare gesammelt und untersucht. 3 ㅇ Exemplare waren mit gut entwickelten Oostegiten mit bzw. 7, 8 und 13 juvenilen Exemplaren.

Nach Angaben des Kollegen B. Russev ist die Temperatur des Wassers $21^{\circ} \mathrm{C}, \mathrm{pH} \mathrm{7,5}$, die Gesamtwasserhärte 14,56 dH (nach der Methode von Boutron und Boude bestimmt). In der Quelle wurden außer $N$. toplicensis auch Tiere folgender Gruppen gefunden: Planaria, Oligochaeta, Hirudinea (Erpobdella), Isopoda (Asellus), Larven von Ephemeroptera (Baetis), Trichoptera und Diptera, Coleoptera (Elminthidae).

\section{ZUSAMMENFASSUNG}

Der Autor beschreibt eine neue Art Niphargus: N. toplicensis n. sp. Die Art ist in der Quelle ,,Toplitzata" bei dem Dorf Mussomischta (Bezirk Goze Delcev) gefunden. N. toplicensis n. sp. zeigt eine Ähnlichkeit mit N. aquilex 
moldavicus Dobreanu, Manolache und Puscariu, 1953, N. smederesanus Karaman, 1950, N. anatolicus Karaman, 1950, N. pancici Karaman, 1929, N. pancici olkanovi Karaman, 1959, doch unterscheidet sie sich durch einige Merkmale (z.B. durch die reichere Bestachelung der Innenlade der I. Maxille, die längeren als breiten Coxalplatten, die Ausbildung und die Aufrüstung des Telsons und des I. Uropods u.a.). Deswegen kann $N$. toplicensis n. sp. zu keiner der obengenannten Arten gezählt werden.

\section{RÉSUMÉ}

L'auteur décrit une espèce nouvelle - Niphargus toplicensis n. sp. de la source "Toplitzata» près du village Mussomischta (district de Goze Delcev). $N$. toplicensis $\mathrm{n}$. sp. est proche des $N$. aquilex moldavicus Dobreanu, Manolache und Puscariu, 1953, N. smederevanus Karaman, 1950, N. anatolicus Karaman, 1950, N. pancici Karaman, 1929, N. pancici olkanosi Karaman, 1959, mais en diffère par quelques caractères (un nombre plus grand des soies sur le lobe interne de la I. maxille, les plaques coxales encore plus longues que larges; la forme et l'armement différentes du telson et des I. uropodes etc.).

\section{LITERATUR}

Carausu, S., Dobreanu, E., und Manolache, C. (1955) - Fauna Republicii Populare Romine. Crustacea, Amphipoda, vol. IV, fasc. 4.

Karaman, St. (1929) - Beiträge zur Kenntnis der Amphipoden Jugoslawiens. Zool. Anz., Bd. 85, Heft 9/10: 217-225.

- (1943) - Unterirdische Amphipoden Südserbiens. Serbisch-Königliche Akademie, t. CXXXV: 251-258.

- (1950) - N. smederevanus n. sp. aus Nordserbien. Serbische Akademie d. Wiss., t. CLXIII, $\mathrm{n}^{0} 2:$ 1-9.

- (1950) - Die Amphipoden Kleinasiens. I. Serbische Akademie d. Wiss., t. CLXIII, $\mathrm{n}^{0} 2: 33-45$.

Karaman, St., und Karaman, G. (1959) - Beitrag zur Kenntnis der Niphargiden Bulgariens. Acta Musei Macedonici scientiarum Naturalium, t. VI, $\mathrm{n}^{0} 7$ (59): 143-162.

Sahellenberg, A. (1942) - Die Tierwelt Deutschlands. 40. Teil. Krebstiere oder Crustacea. IV. Flohkrebs oder Amphipoda. 

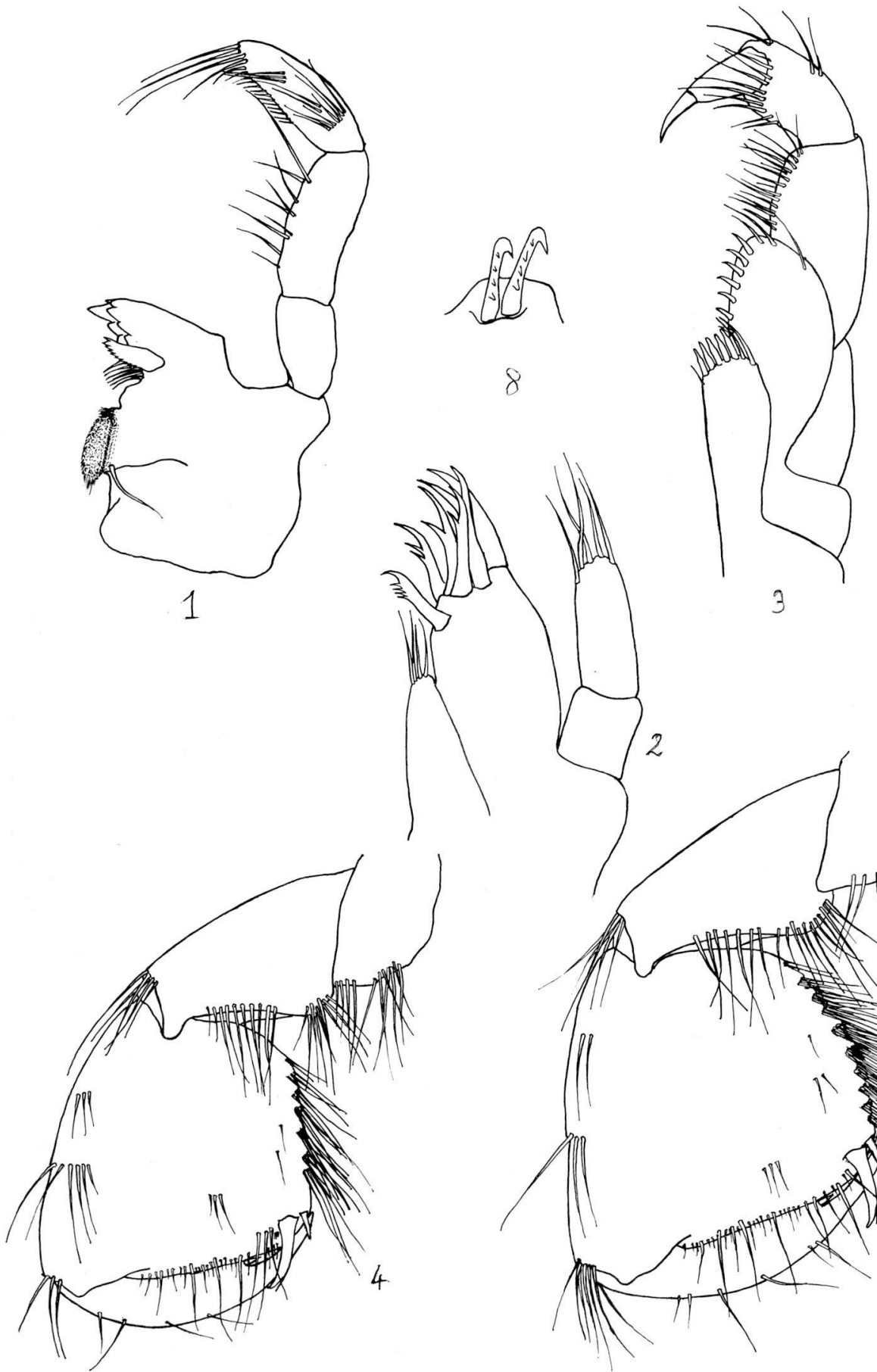
SPELEOLOGY II
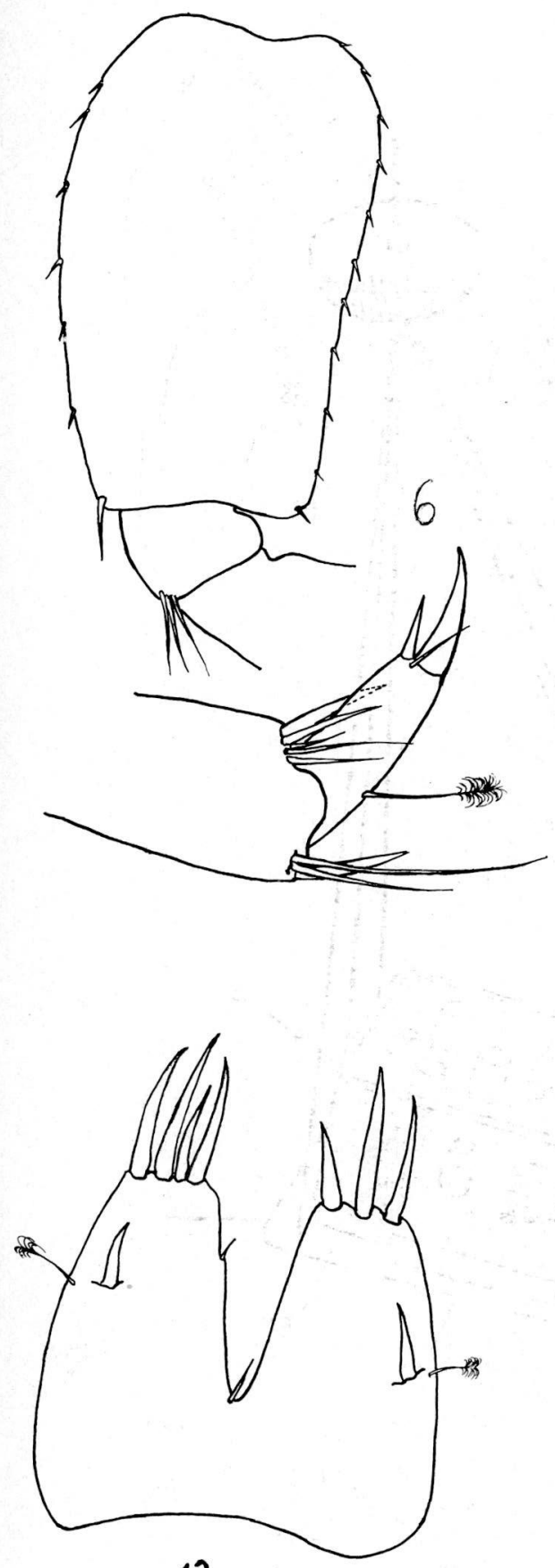

13
(Andreev, 2)

PLATE 54

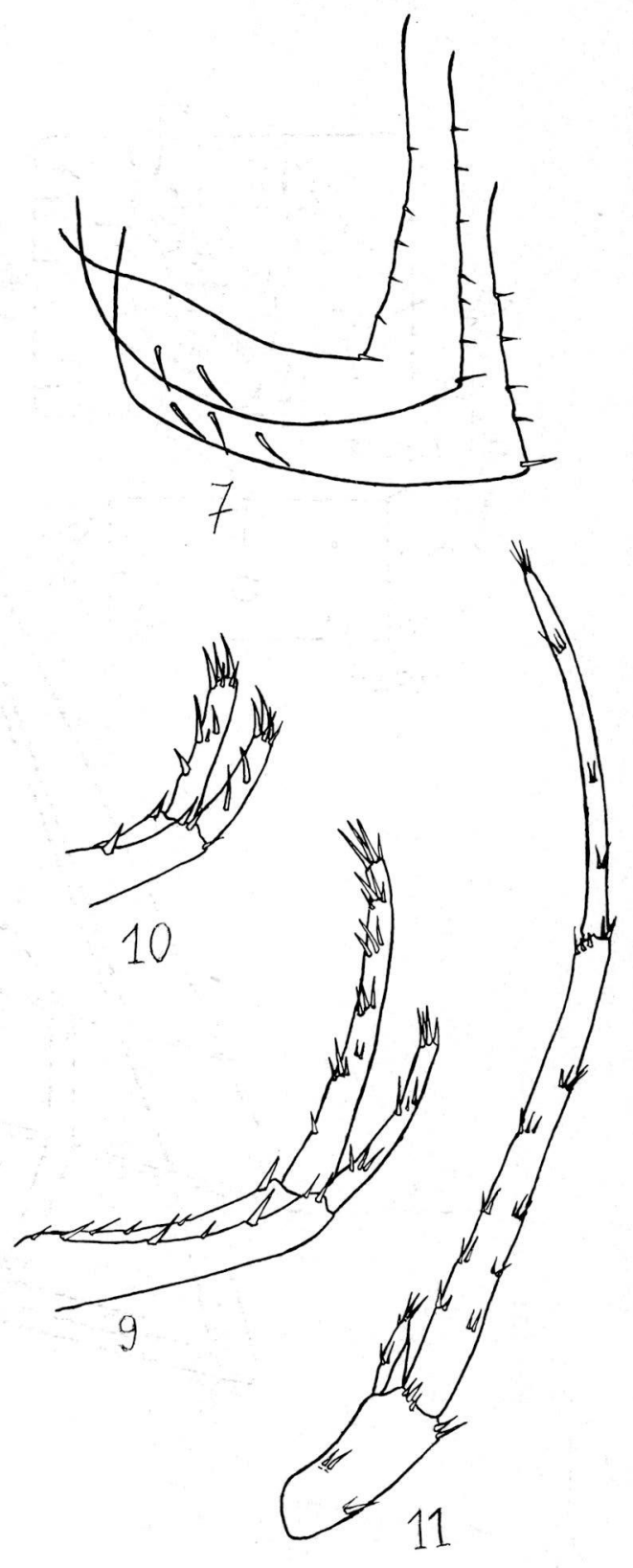

